Paper presented at the Third Workshop on Radiation-Induced and/or Process-Related Electrically Active Defects in SemiconductorInsulator Systems, Research Triangle Park, NC, September 10-13, 1991 $\operatorname{cou} 6-9101329+2$

BNL -46198

(Rev. 1/92)

\title{
FAST NEUTRON RADIATION DAMAGE EFFECTS ON HIGH RESISTIVITY SILICON JUNCTION DETECTORS*
}

\author{
Zheng $\mathrm{Li}$ and $\mathrm{H}$. W. Kraner
}

Brookhaven National Laboratory, Upton, NY 11973

January 1992

*This research was supported by the U. S. Department of Energy: Contract No. DE-AC02-76CH00016. 


\title{
FAST NEUTRON RADIATION DAMAGE EFFECTS ON HGH RESISTIVITY SILICON JUNCTION DETECTORS*
}

\author{
Zheng $\mathrm{Li}$ and H. W. Kraner \\ Brookhaven National Laboratory, \\ Instrumentation Division, 535-B \\ Upton, NY 11973 \\ 516-282-7604
}

BNL- $-46198-$ Rev 。

DE92 010131

\begin{abstract}
$\mathrm{p}^{+}-\mathrm{n}^{-}-\mathrm{n}^{+}$silicon radiation detectors made of high resistivity Si material $(\rho \geq 2 \mathrm{k} \Omega$-cm) were irradiated to a neutron fluence of a few times of $10^{13} \mathrm{n} / \mathrm{cm}^{2}$. Dependence of detector leakage current, reverse bias capacitance, and effective doping concentration of the Si substrate on the neutron fluence have been systematically studied. It has been found that the detector leakage current increases linearly with neutron fluence in the range studies, with a damage constant of $\alpha$ $=9 \times 10^{-17} \mathrm{~A} / \mathrm{cm}\left(\Delta \mathrm{I}=\alpha \mathrm{V} \Delta \phi_{\mathrm{n}}\right)$, and the $\mathrm{C}-\mathrm{V}$ characteristics of detectors irradiated to $\phi_{\mathrm{n}}>$ $10^{12} \mathrm{n} / \mathrm{cm}^{2}$ become frequency dependent. Models using several defect levels in the band gap are proposed to describe the frequency dependent $\mathrm{C}-\mathrm{V}$ effects and the electrical field profile after high neutron fluence irradiation.
\end{abstract}

\section{INTRODUCTION}

Particle detectors made from high resistivity silicon are now widely used in high energy physics community. The most common detector configuration is the $\mathrm{p}^{+}-n^{-}-n^{+}$implanted junction diode device. For a $300 \mu$ m thick detector fully depleted at a reverse bias between $30 \mathrm{~V}$ to $150 \mathrm{~V}$, the iesistivity of the n-type Si must be between $2 \mathrm{k}$ to $10 \mathrm{k} \Omega-\mathrm{cm}$, which leads to a net doping concentration of less than $2 \times 10^{12} / \mathrm{cm}^{3}$. Displacement damage caused by fast neutrons $(E>100 \mathrm{keV}$ ) has been ، major concern for high resistivity silicon detectors working in the high radiation environment anticipated for the Superconductin ${ }_{4}$ Super Collider (SSC) and the Large Hadron Collider (LHC), where the annual fluence of fast neutrons can be as high as $10^{13} \mathrm{n} / \mathrm{cm}^{2}$. At this neutron fluence, concentrations of various defects in the band gap can be close to or exceed the net doping concentration, causing problems unexpected in low resistivity silicon materials. In this work, effects of fast neutron radiation (up to the fluence of a few times of $10^{13} \mathrm{n} / \mathrm{cm}^{2}$ ) on the electrical properties of high resistivity silicon detectors, such as leakage current, capacitance-voltage $(C-V)$ characteristics, and possible type inversion $(n \rightarrow p)$ have been systematically studied.

\section{EXPERIMENTAL}

Fast neutrons from $10 \mathrm{keV}$ to $2.2 \mathrm{MeV}$ with $\bar{E}=1 \mathrm{MeV}$ were obtained from the ${ }^{7} \mathrm{Li}(\mathrm{p}, \mathrm{n})$ reaction using $4 \mathrm{MeV}$ protons from a van de Graaff accelerator at the University of Lowell. The $p^{+}-n^{-}-n^{+}$ion-implant junction detectors used in this study were made on n-type $\langle 111\rangle \mathrm{Si}$ wafers from Wacker, with resistivities ranging from $2 \mathrm{k} \Omega$-cm to $10 \mathrm{k} \Omega$-cm. Si wafers were given standard RCA cleaning before being oxidized to about $4500 \AA \mathrm{SiO}_{2}$ at $1100^{\circ} \mathrm{C}$. The typical value of minority carrier life time obtained from C-t measurement is above $1 \mathrm{msec}$ after oxidation, and the detector leakage currents are between 4-20 nA/cm²/300 $\mu \mathrm{m}$. A multi-frequency HP 4192 LF Impedance Analyzer was used for capacitance-voltage $(C-V)$ measurements at various frequencies. All C-V measurements were performed with the HP 4192 LF Impedance Analyzer in a series mode to take into account the high series resistance $\left(\mathrm{R}_{\mathrm{s}} \geq 1 \mathrm{k} \Omega\right)$.

Keywords: Neutron radiation damage, radiation detector, high resistivity silicon, frequency-dependent capacitance, donor ro noval and/or compensation, type-inversion, electrical field distribution

*This research was supported by the U. S. Department of Energy: Contract No. DE-AC02-76CH00016.

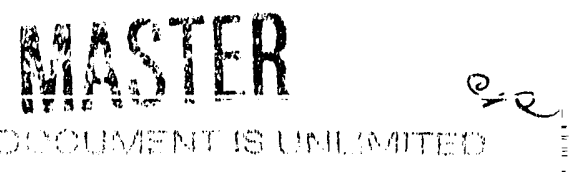




\section{RESULTS AND DISCUSSIONS}

The detector leakage current $(I)$ has been found to increase linearly with the neutron fluence $\phi_{n}$ up to $10^{14} \mathrm{n} / \mathrm{cm}^{2}$ due to degradation of minority carrier lifetime. A damage coefficient $\alpha$, defined as $\Delta l=\alpha V \phi_{n}$ with $V$ being the depleted volume and $\phi_{n}$ being the neutron fluence $\left(\mathrm{n} / \mathrm{cm}^{2}\right)$, can be determined from experimental data. Data shown in Fig. 1 result in $\alpha=9.48 \times 10^{-17} \mathrm{~A} / \mathrm{cm}$ at $24^{\circ} \mathrm{C}$. A consensus value of $\alpha=8 \times 10^{-17} \mathrm{~A} / \mathrm{cm}$ is realized for $20^{\circ} \mathrm{C}$ and $1 \mathrm{MeV}$ Kemmer corrected.

For detectors irradiated to high neutron fluence $\left(\phi_{n}>10^{12} / \mathrm{cm}^{2}\right)$, the C-V characteristics become frequency dependent as it is shown in Fig. 2. A model using two acceptor-like trap levels shown in Fig. 3 has been developed to describe the frequency dependence and dopant compensation effects.[1] In this model, the effective doping density, $N_{e f f}$ is compensated by the somewhat shallow acceptor-like level $E_{t 2}$, i.e.,

$$
\begin{gathered}
N_{e f f}=N_{D}^{0}-N_{A}^{0}-N_{t 2} \\
\text { with } N_{t 2}=\gamma \phi_{n}
\end{gathered}
$$

where $\gamma$ is the introductory rate of $N_{t 2}$.

Donor removal is known to occur due to the formation of E-center, P-V (phosphorus-vacancy complex), that removes the phosphorus from the substitution site. This level can be adjusted so that it does not play a significant role in the frequency dependence but it can reasonably be there to provide the observed donor compensation.

$$
d N_{D}=-\beta N_{D} d \phi_{n}
$$

or

$$
N_{D}=N_{D}^{o} e^{-\beta \phi_{n}}
$$

where $\beta$ is the donor removal rate.

Equation (1) therefore can be modified as

$$
N_{e f f}=N_{D}^{0} e^{-\beta \phi n}-N_{A}^{0}-\gamma \phi_{n}
$$

Figure 4 shows $C(\omega, V)$ curves calculated from the model. The calculation parameters are listed in the figure. A good agreement between the experiment data and calculation is illustrated in Fig. 5. As it is shown in Figs. 3 and 4 , the occupancy of level $E_{t l}$ is controlled by bias and it contributes to the frequency dependence of capacitance. On the other hand the level $E_{t 2}$ has little to do with the bias and it contributes to compensation effect shown in Fig. $2 b$, where the full depletion voltage $V_{d}$ has been observed to decrease from $55 \mathrm{~V}$ before n-radiation to about $20 \mathrm{~V}$ after $7.8 \times 10^{12} \mathrm{n} / \mathrm{cm}^{2}$ radiation. Our DLTS work has shown that $E_{t l}$ may be the E-center $\left(E_{c}-0.4 \mathrm{eV}\right)$ and the $E_{t 2}$ may be a level at $E_{c} 0.55 \mathrm{eV}$, possibly a double vacancy $(\mathrm{V}-\mathrm{V})$ at a different charge state [2]. Since all solutions are in their analytical forms [1], it gives physical insights more directly than those derived from numerical models $[3,4]$. There are other models in the literature that do not explain the effect of voltage-independence of capacitance measured at high frequencies $[4-8]$.

When the neutron fluence $\phi_{n}$ is close to or greater than $10^{13} \mathrm{n} / \mathrm{cm}^{2}$, the defect concentrations can be close to or exceed the dopant concentration $\left(10^{12} / \mathrm{cm}^{3}\right)$. This could lead to so called type-inversion $\left(\mathrm{n}^{-} \rightarrow \mathrm{p}\right)$ and possibly cause a basic structural change in the detector as the rectifying junction switches from the $\mathrm{p}^{+}$to the $\mathrm{n}^{+}$contact. This change of type could make some detector configurations, such as the silicon drift chamber detector that uses a potential well created by a $\mathrm{p}^{+}-\mathrm{n}-\mathrm{p}^{+}$structure to collect electrons, not workable [9].

Type inversion, however, has now become an interesting but complicated problem. Contradictory conclusions have been reached by different groups based on different measurements. In this work, MOS capacitor techniques and back-to-back diode techniques have been used. Figure 6 shows the C-V characteristics of MOS capacitors with ntype $\mathrm{Si}$ substrate under various fluence of neutron radiations. It is clear that up to the neutron fluence of $2.15 \times$ $10^{13} \mathrm{n} / \mathrm{cm}^{2}$, the MOS capacitor still exhibits the characteristics of a n-type Si substrate, suggesting no type-inversion. The decrease of $C_{o x}$ at high frequency is caused by the increase of $R_{s}$ with neutron radiation. The increase of $C_{\min }$ 
at $f=10 \mathrm{kHZ}$, on the other hand, is due to the decrease in response time of the minority carrier $\tau_{R}$ with neutron radiation, which is in turn related to minority carrier lifetime $\tau_{p}$ as in the following [10]:

$$
\tau_{R}=\frac{1}{\sqrt{2}}\left(\frac{N_{D}}{n_{i}}\right)\left(\tau_{T_{n}} \tau_{T p}\right)^{1 / 2}\left(1-\frac{v_{t}}{U_{B}}\right)^{1 / 2}
$$

where $U_{B}=E_{F}-E_{i}$ and $v_{t}=E_{t}-E_{i}$

Since the transition frequency for minority carrier to respond to the measurement is proportional to $1 / \tau_{R}$, i.e.:

$$
f_{t} \propto \frac{1}{\tau_{R}}
$$

One would expect $f_{t}$ to go up with the neutron fluence as it is shown in Fig. 6. Since there is minimum ionization radiation during the neutron exposure (significant flat band voltage shifts of MOS structures were not observed), the damage to the oxide and to the $\mathrm{SiO}_{2} / \mathrm{Si}$ interface are minimum and are not of concern here.

Back-to-back diode I-V characteristics shown in Figs. 7 and 8 also indicate that the junction is still in the front, i.e., still $\mathrm{p}^{+}-\mathrm{n}$ junction for the neutron fluence to above $1.2 \times 10^{13} \mathrm{n} / \mathrm{cm}^{2}$.

However, Lindstroem et al., [11], using an $\alpha-$ source $(E=5 \mathrm{MeV})$ that has a range of $20 \mu \mathrm{m}$ in Si and penetrates both $\mathrm{p}^{+}$and $\mathrm{n}^{+}$contacts have found in their charge collection experiments that more charge has been collected when the $\alpha$-source was placed in the back of the detector than that when the $\alpha$-source was placed in the front when a high resistivity $(\rho \geq 5 \mathrm{k} \Omega-\mathrm{cm}) \mathrm{p}^{+}-\mathrm{n}^{-}-\mathrm{n}^{+}$detector was irradiated to above $8 \times 10^{12} \mathrm{n} / \mathrm{cm}^{2}$. This result indicates more electrical field in the back than that in the front and a shift of junction from front to back or type-inversion was suggested. Our data of $\alpha$-particle induced current pulse $(I / t))$ measurement on detectors irradiated also to above $1 \times 10^{13} \mathrm{u} / \mathrm{cm}^{2}$ also show high field on the back of the detector. However, a similar high field has also shown existed on the front side, indicating junctions may be on both front and back side of the detector but with reduced charge collection (see Fig. 9). Our resistivity data (Fig. 10) through series resistance $\left(R_{s}\right)$ value of the C-V measurement show that detector substrate resistivity saturates between $6 \times 10^{12}$ to $1.5 \times 10^{13} \mathrm{n} / \mathrm{cm}^{2}$ indicating an intrinsic or near intrinsic bulk instead of inversion. Data of our measurement of resistivity on a $n^{+} / n^{n} / n^{+}$resistor also show the resistivity saturates at a value of $255 \mathrm{k} \Omega-\mathrm{cm}$ at $\sim 1.5 \times 10^{13} \mathrm{n} / \mathrm{cm}^{2}$. The relatively low value of saturation fluence is due to the fact that the starting material is itself high resistivity or near intrinsic $\left(N_{D}<\right.$ $10^{12} / \mathrm{cm}^{2}$ ). Based on these observations, a model of $\mathrm{p}^{+} / \mathrm{n} / \mathrm{i} / \mathrm{p} / \mathrm{n}^{+}$structure is proposed in this study. As it is shown in Fig. 11, at a meaningful operation bias ( $\mathrm{V} \leq 200$ volts), a donor-like level(s) on the $n$-side and an acceptor-like level(s) on the p-side are controlling the band bending and therefore the field in the structure with little or no E-field in the bulk. Free carriers created hy $\alpha$-particle travel in the E-field and get trapped by shallow traps in the low or no E-field bulk before being collected. This model can explain the MOS C-V data, back-to-back diode data and $\alpha$-source results. Unfortunately, should further measurements at higher fluences indicate this effect significantly reduces the internal electrical field in a junction detector, providing charge collection only near the contacts, the operation of such detectors in high neutron fluences will be severely challenged.

In conclusion, changes of electrical properties of high resistivity Si detectors due to fast neutron radiation have been studied up to fluences of several $\times 10^{13} \mathrm{n} / \mathrm{cm}^{2}$. At low fluences, $\phi_{n}<10^{12} \mathrm{n} / \mathrm{cm}^{2}$, the Shockley-Read-Hall generation and recombination process via various defect levels is the dominant mechanism that contributes to the increase of detector leakage current (or decrease of minority carrier lifetime). At intermediate fluences, $10^{12} \mathrm{n} / \mathrm{cm}^{2}$ $\leq \phi_{n}<10^{13} \mathrm{n} / \mathrm{cm}^{2}$, donor-removal becomes important and concentrations of acceptor-like defect levels are high enough to affect detector $\mathrm{C}-\mathrm{V}$ characteristics. At high fluences, $\phi_{n} \geq 10^{13} \mathrm{n} / \mathrm{cm}^{2}$, the Si bulk may become intrinsic or near intrinsic and donor-like defect level(s) and acceptor-like defect level(s) may become dominant to control the electrical field in the front side and hack side of the detector, respectively. 


\section{REFERENCES}

1. Zheng Li and H. W. Kraner, IEEE Trans, on Nucl. Sci. 38, 244 (1991).

2. Zheng Li, Wei Chen, and H. W. Kraner, Nucl. Instrum. Methods A397, 585 (1991).

3. E. P. Eernisse, Phys. Lett. 18 (5), 183-186, (1971).

4. Y. Zohta, J. Appl. Phys. 43 (4), 1713-1716, (1972).

5. Y. Zohta and Y. Ohmura, Appl. Phys. Lett. 21 (3), 117-119, (1972).

6. G. Vincent, D. Bois, and P. Pinard, J. Appl. Phys. 46 (12), 5173-5178, (1975).

7. Y. Tokuda and A. Usami, J. Appl. Phys. 48 (4), 1668-1672, (1977).

8. Y. Tokuda and A. Usami, J. Appl. Phys. 49 (2), 603-607, (1978).

9. E. Gatti and P. Rehak, Nucl. Instrum. Methods 225, 608 (1984).

10. E. H. Nicollian and J. R. Brews, MOS (Metal Oxide Semiconductor) Physics and Technology, (John Wiley \& Sons, New York), p. 139 (1982).

10. G. Lindstroem, et al., Calorimetry in High Energy Physics, D. F. Anderson, et al., eds., World Scientific Press, Singapore (1991), p. 467. 


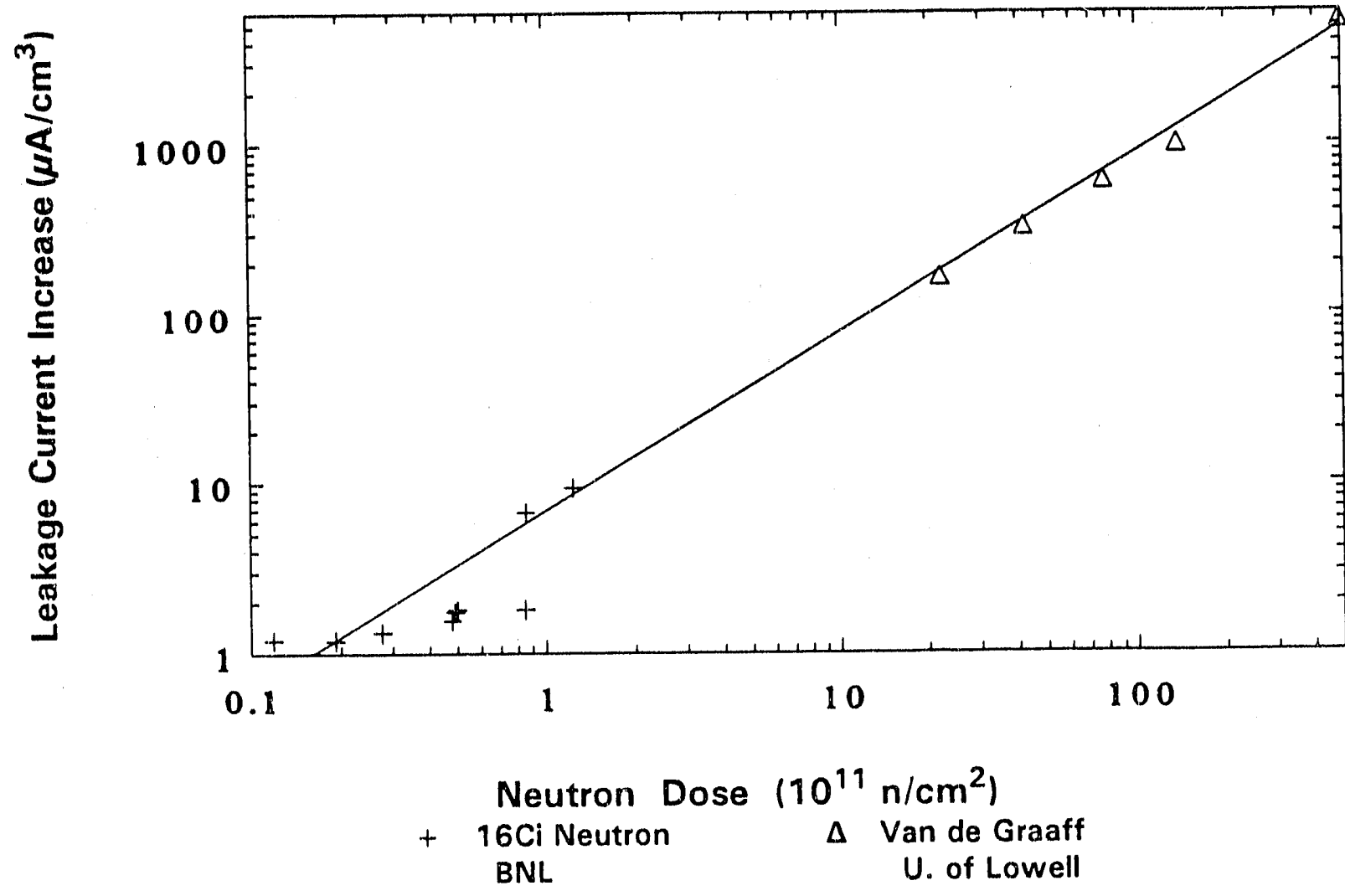

Fig. 1. Increase of detector volume leakago current with fast neutron fluence up to $5 \times 10^{13} \mathrm{n} / \mathrm{cm}^{2}$. A linear fit is also shown. 


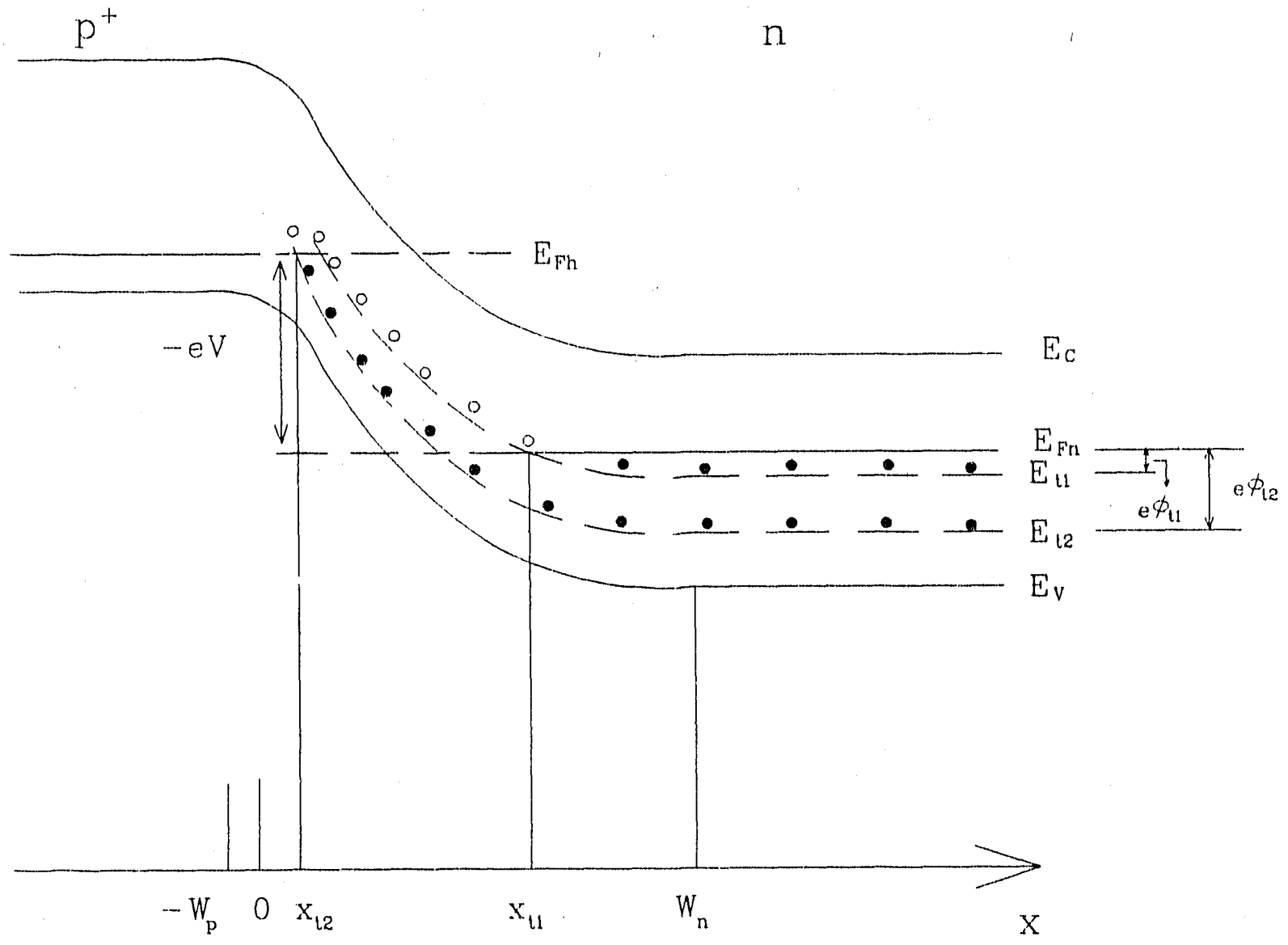

Fig. 3. Energy band diagram for the proposed two-level model describing the observed frequency-dependent $C-V$ effects. 


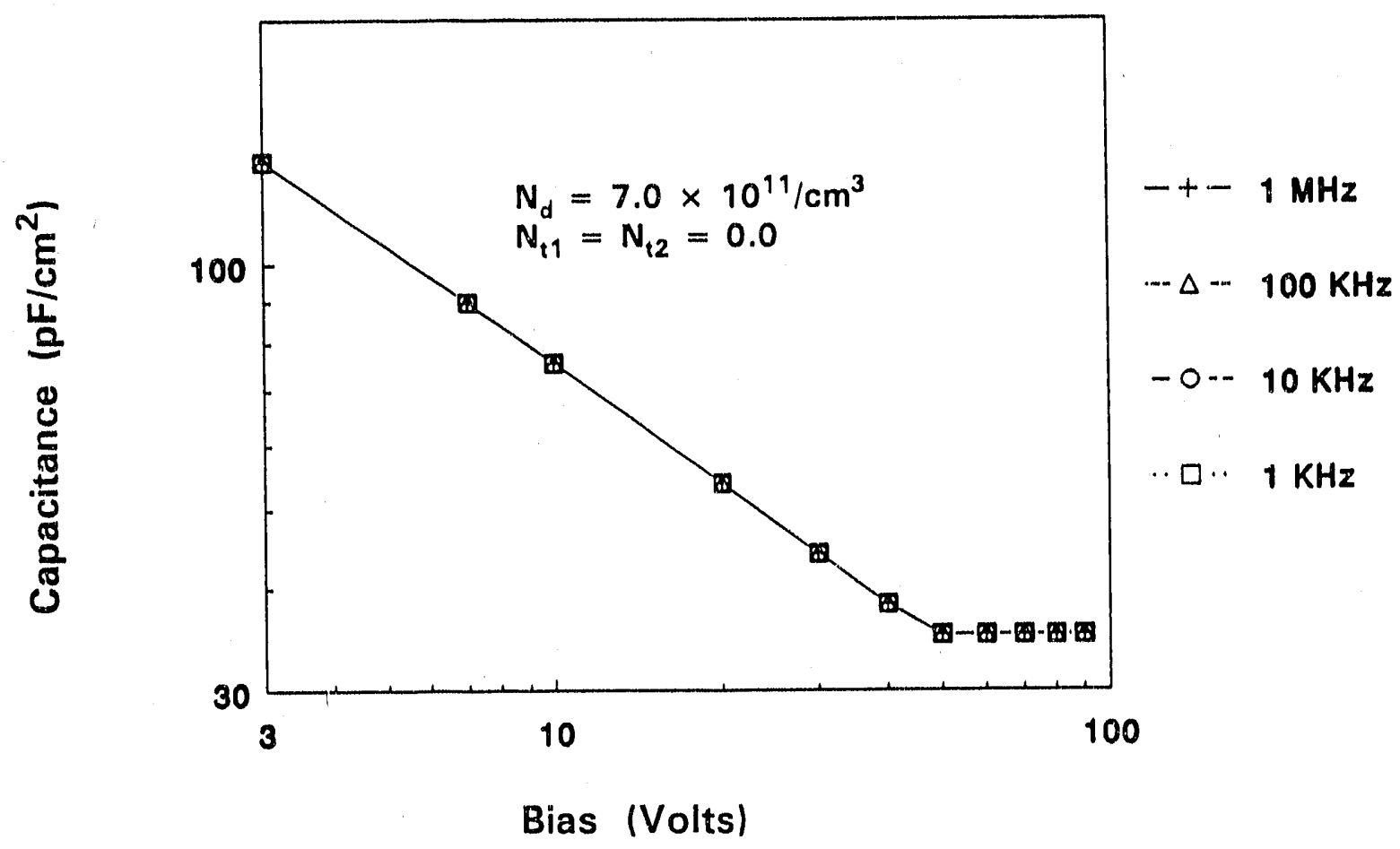

a) No defect (control)

$-+-1 \mathrm{MHz}$

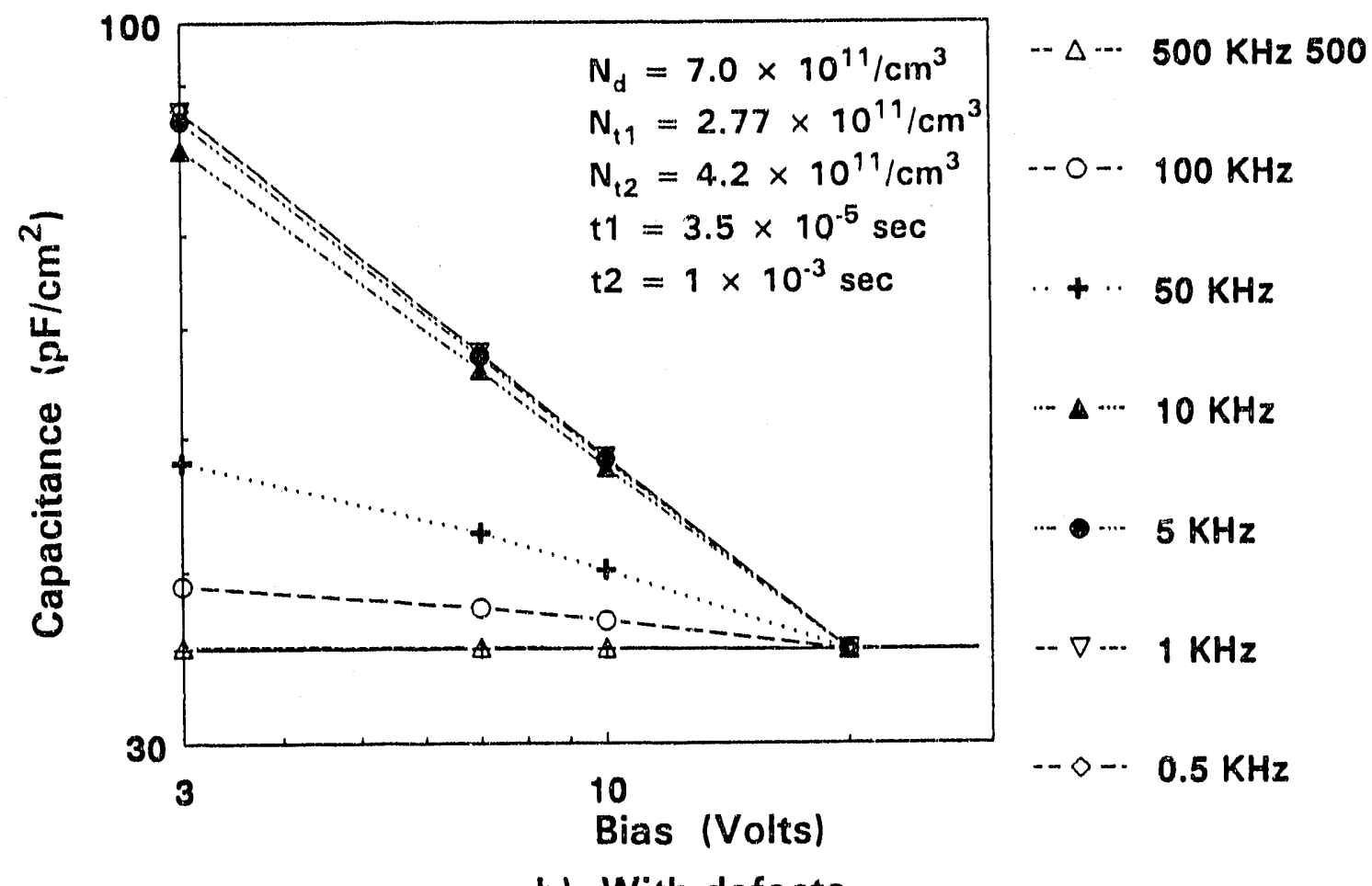

b) With defects

Fig. 4. Calculated frequency-dependent C-V characteristics with a) No defects (control); and b) two defect levels. 


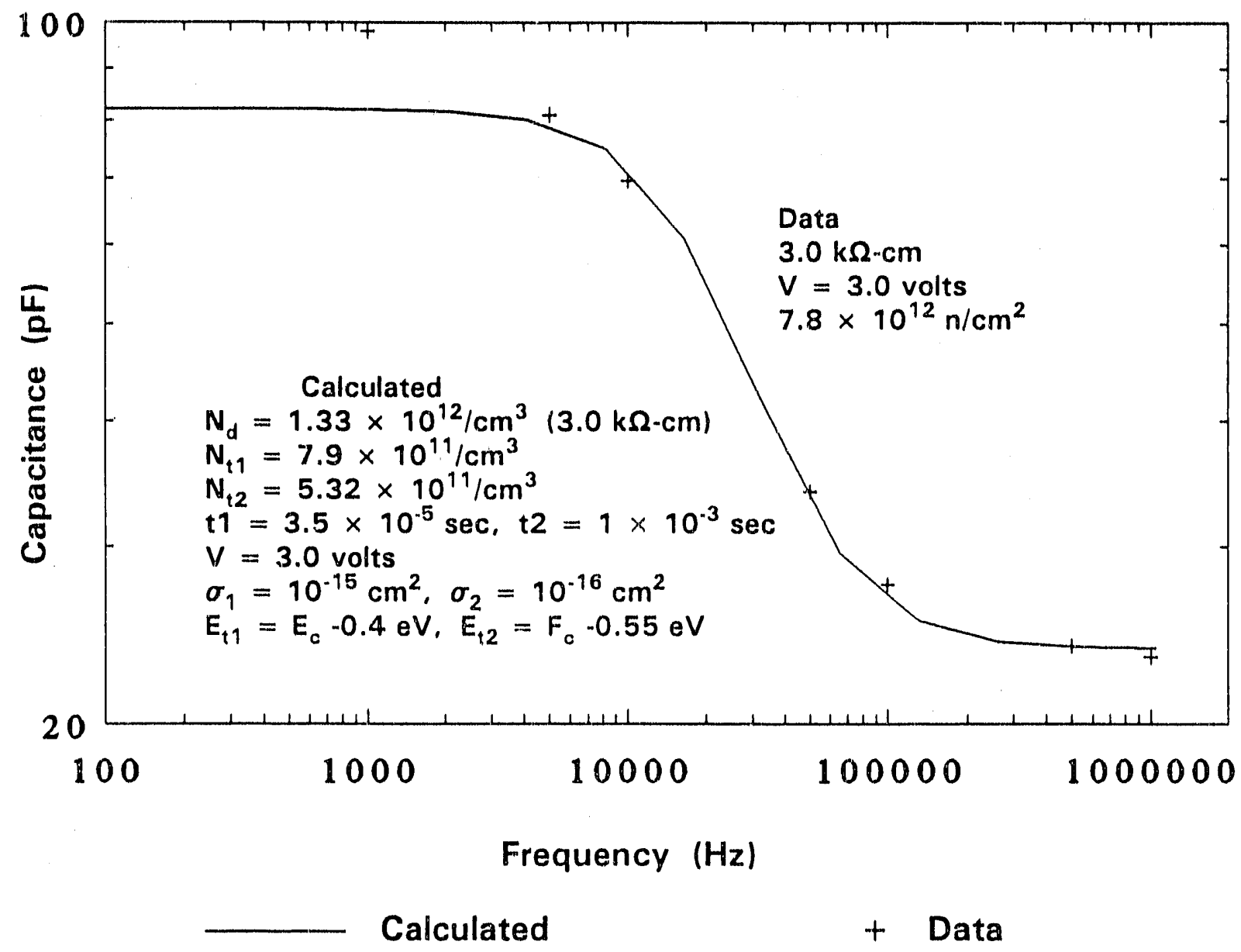

Fig. 5. Comparison between calculated and measured C- $f$ data. 
MOS CAPACITOR UNDER n-RAD.

$\mathrm{n}(111) 4 \mathrm{~K}$ olnm-cm
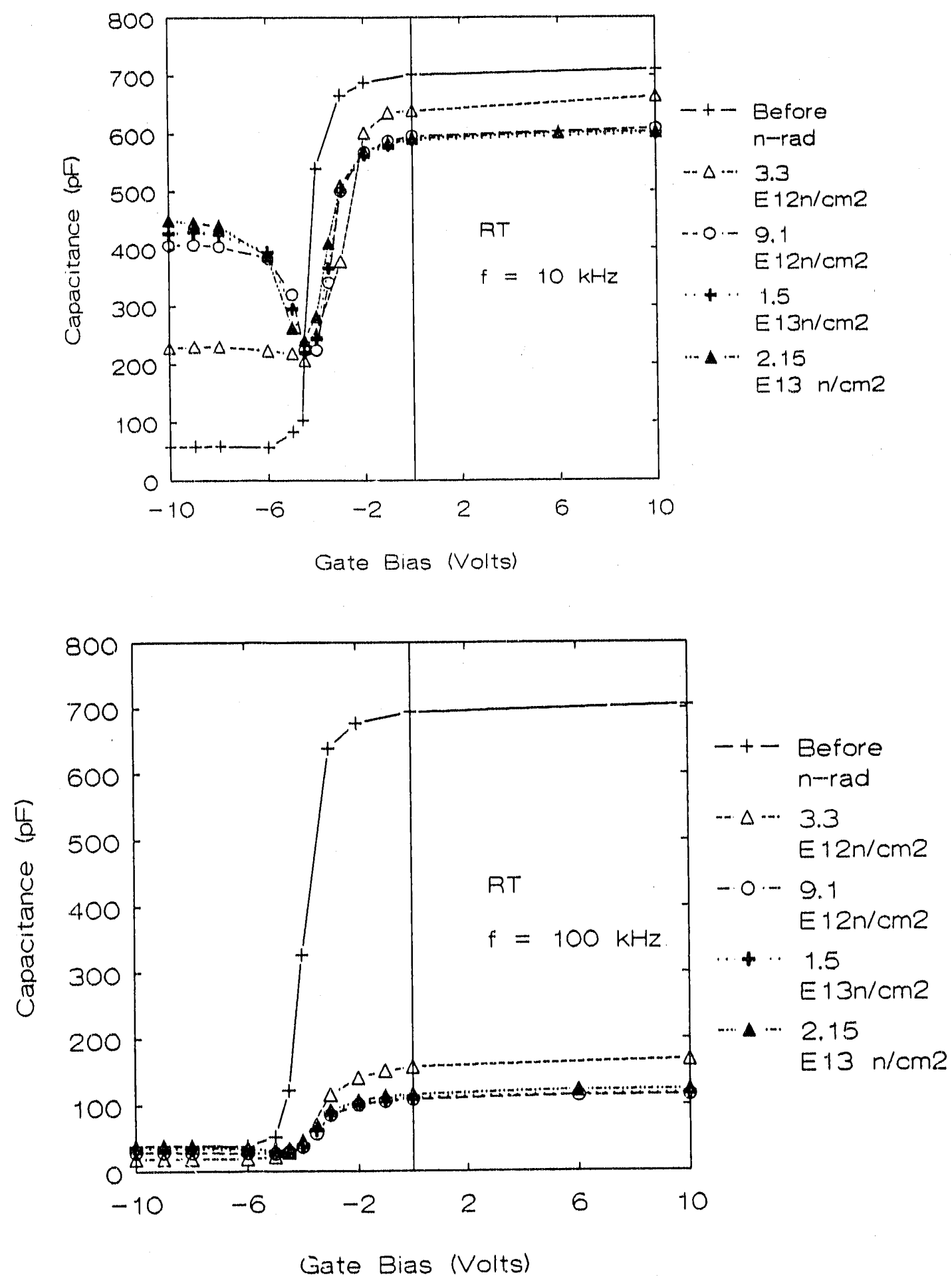

Fig. 6. $\quad \mathrm{C}-\mathrm{V}$ characteristics of an $\mathrm{Al} / \mathrm{SiO}_{2} / \mathrm{n}$-Si MOS capacitor after consecutive neutron radiations up to $2 \times 10^{13} \mathrm{n} / \mathrm{cm}^{2} . \mathrm{SiO}_{2}$ here is OXC (Oxide $\mathrm{C}\left(1100^{\circ}\right)$ ). 
BACK TO BACK DIODE I-V CHARACTERISTICS

$\# 1001-2$ and guard ring, $1.4 E 13 \mathrm{n} / \mathrm{cm} 2$

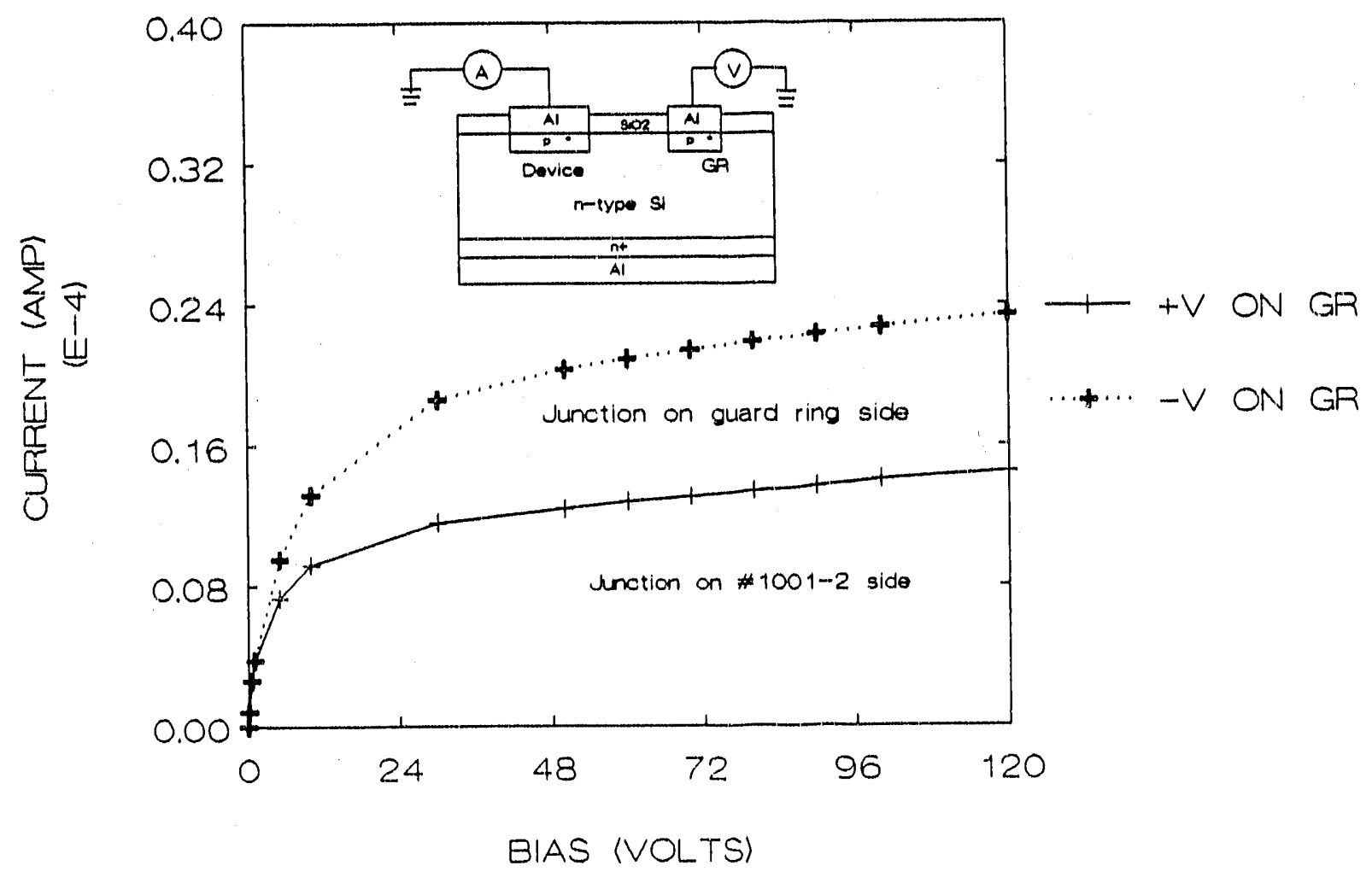

Fig. 7. I-V characteristics of the back-to-back diode configuration after n-radiation. 
BACK TO BACK DIODE I-V CHARACTERISTICS \#194-6, p+-n-SiO2, $n(111), 4 \mathrm{k}$ ohm-cm

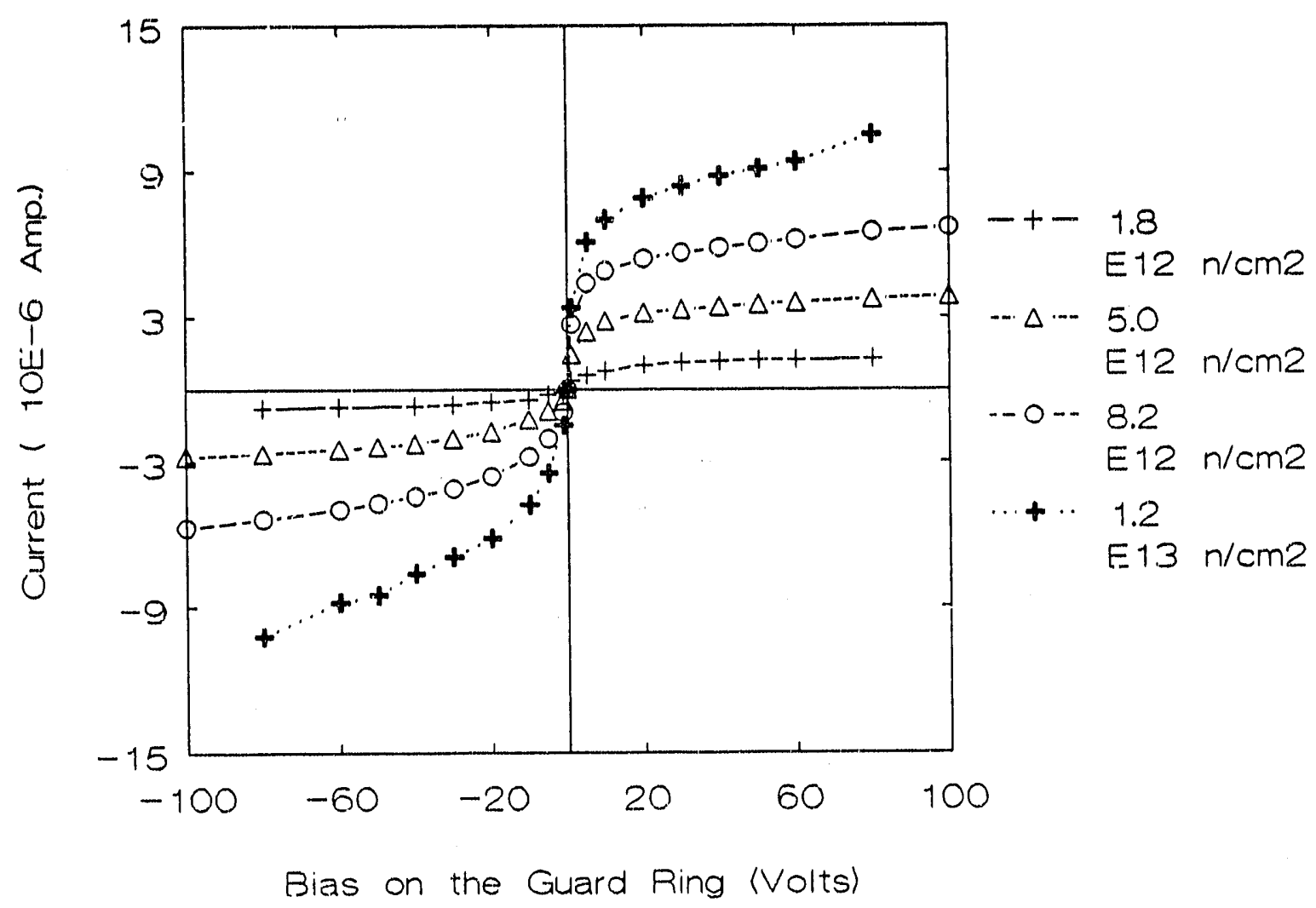

Fig. 8. I-V characteristics of the back-to-back diode configuration with $\mathrm{SiO}_{2}$ on the back side after $n$-radiation. 

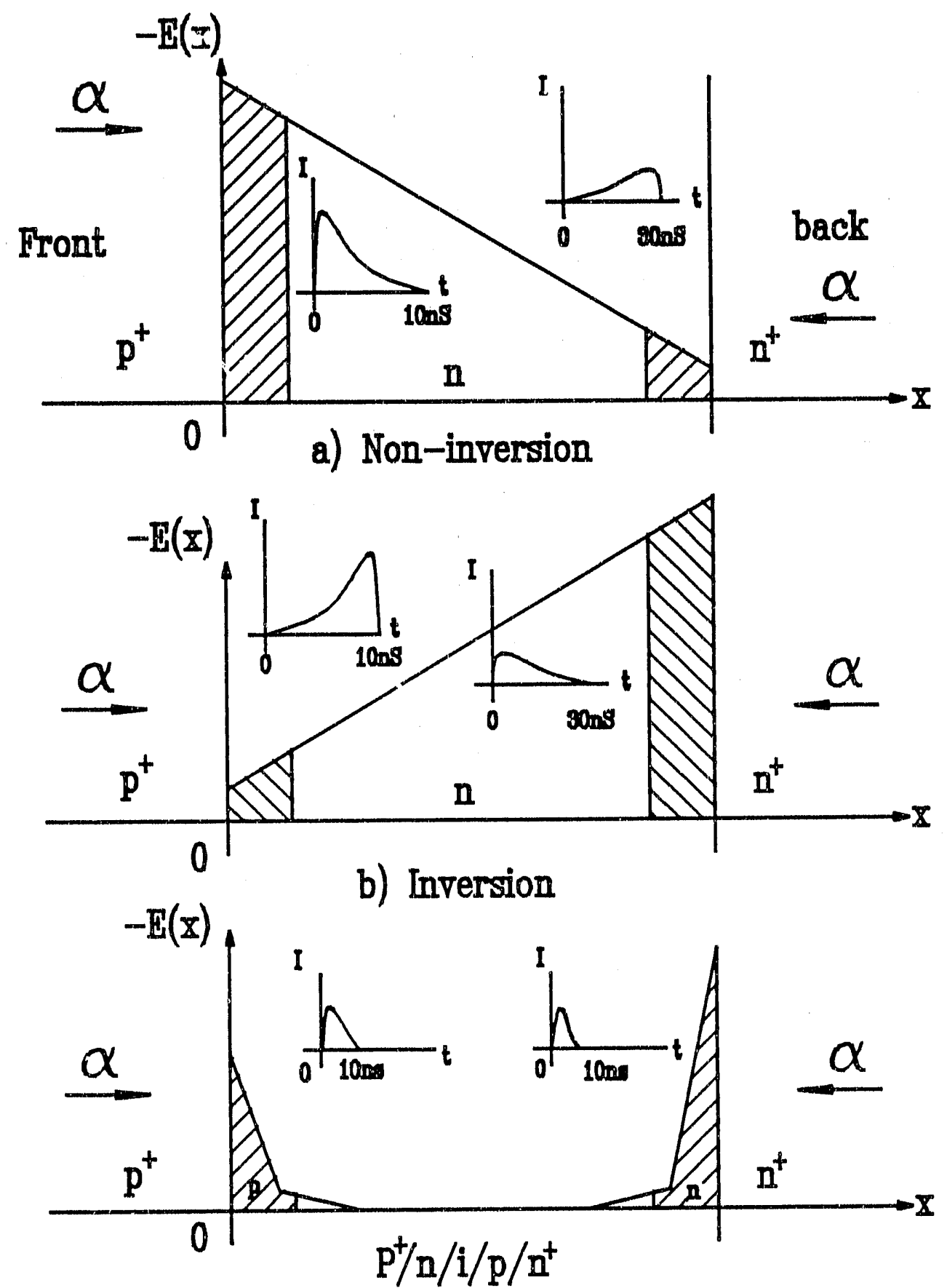

c) Junction on both sides

Fig. 9. Schematic of the electrical field profiles in the Si detector for three different configurations. Corresponding current pulses respond to $a$-particle on the front and back of the detector are also shown. 


\section{MOS SERIES RESISTANCE}

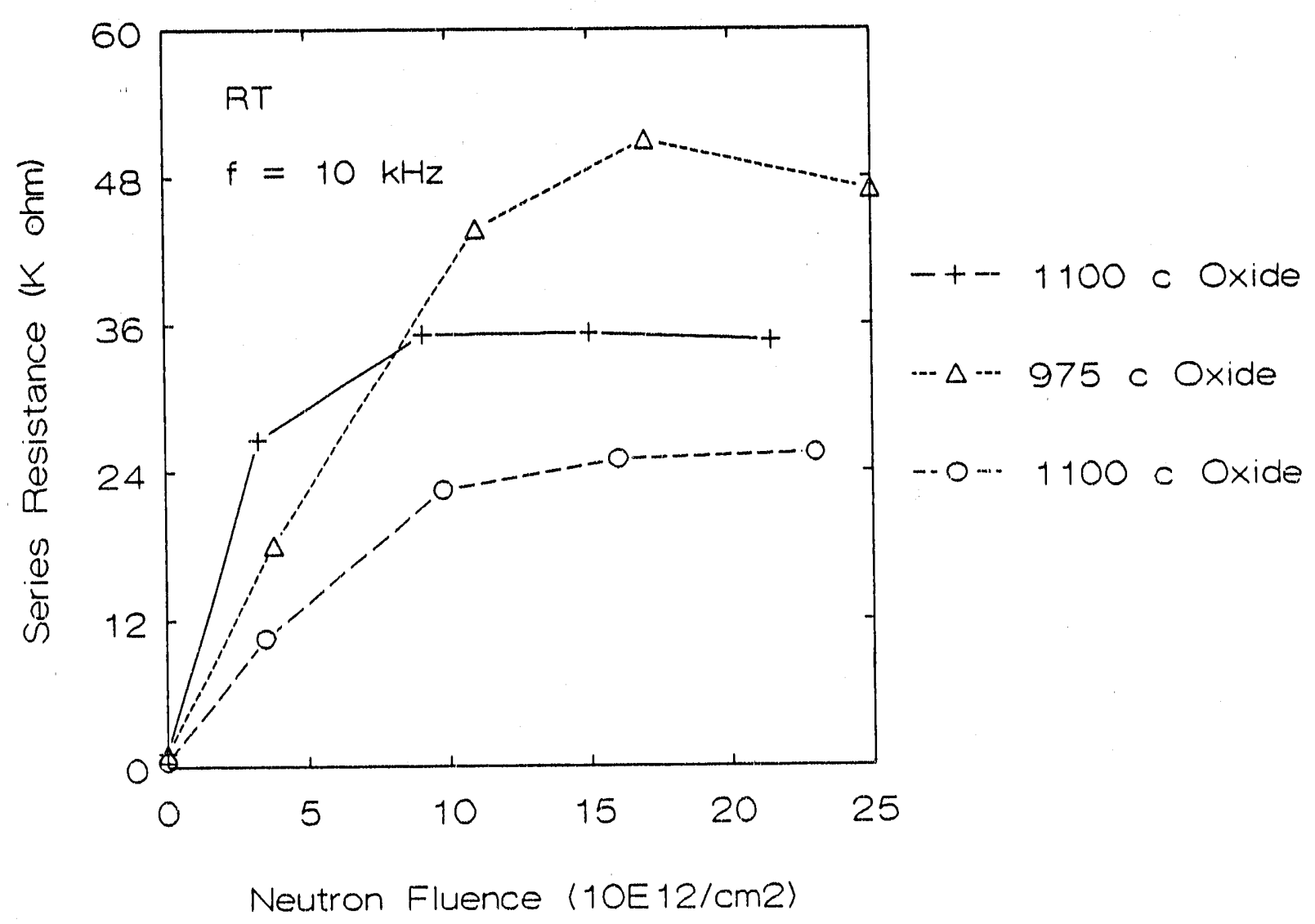

Fig. 10. Series resistance $R_{s}$ of MOS capacitors measurement as a function of neutron fluence. 


$$
\begin{gathered}
v_{A}=v_{A n}+v_{A p} \\
N_{T D}, E_{T D}: \text { Donor type traps } \\
N_{T A}, E_{T A}: \text { Acceptor type traps } \\
N=p \approx n_{i}=1.0 \times 10^{10} / \mathrm{cm}^{3}
\end{gathered}
$$

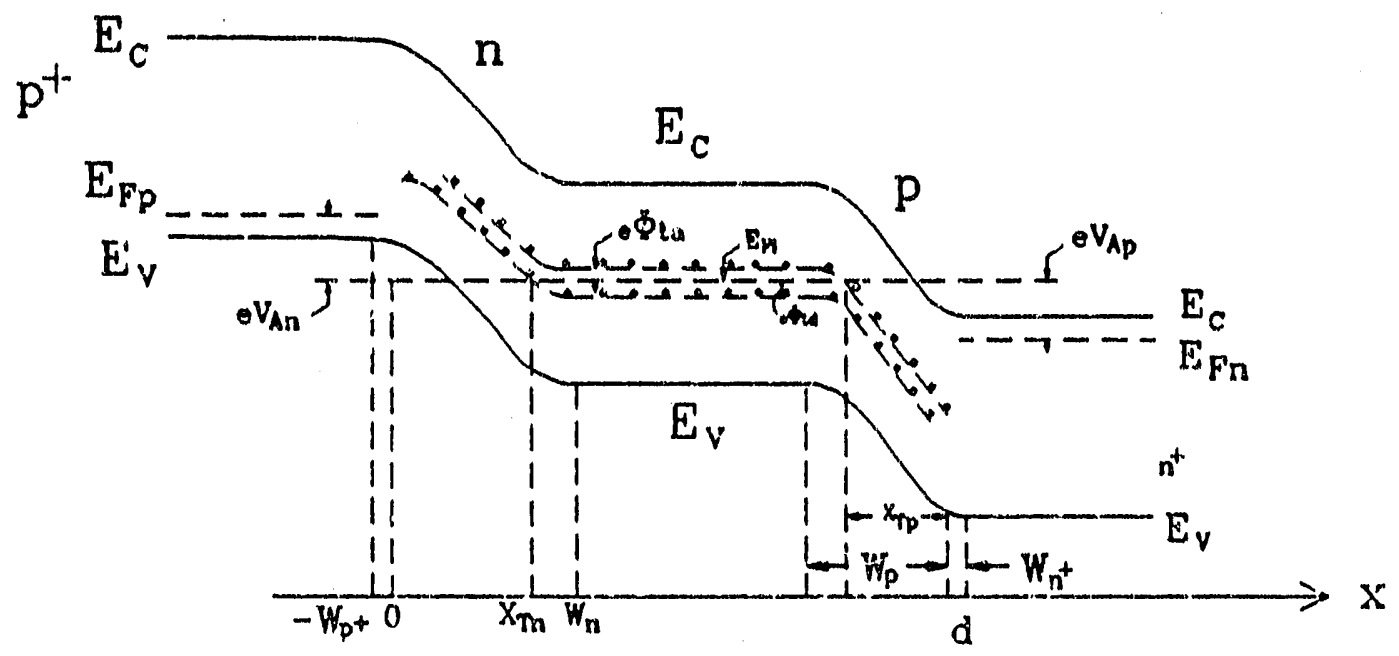

Fig. 11. Proposed $\mathrm{p}^{+}-\mathrm{p}-\mathrm{i}-\mathrm{nn}-\mathrm{n}^{+}$band diagram for a detector irracliated to high neutron fluence $\left(\phi_{n} \geq 10^{13} \mathrm{n} / \mathrm{cm}^{2}\right)$. 

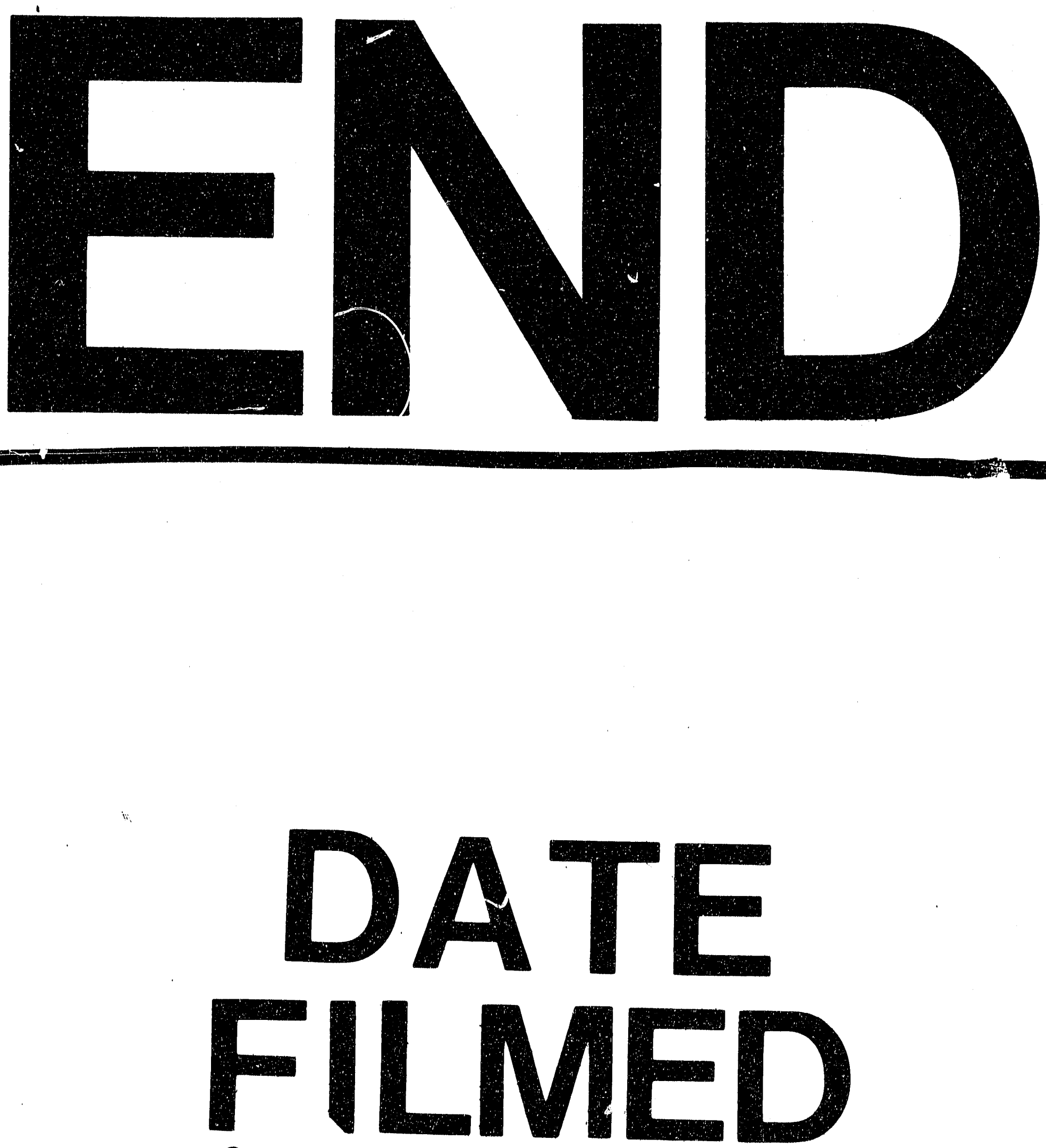

$=$

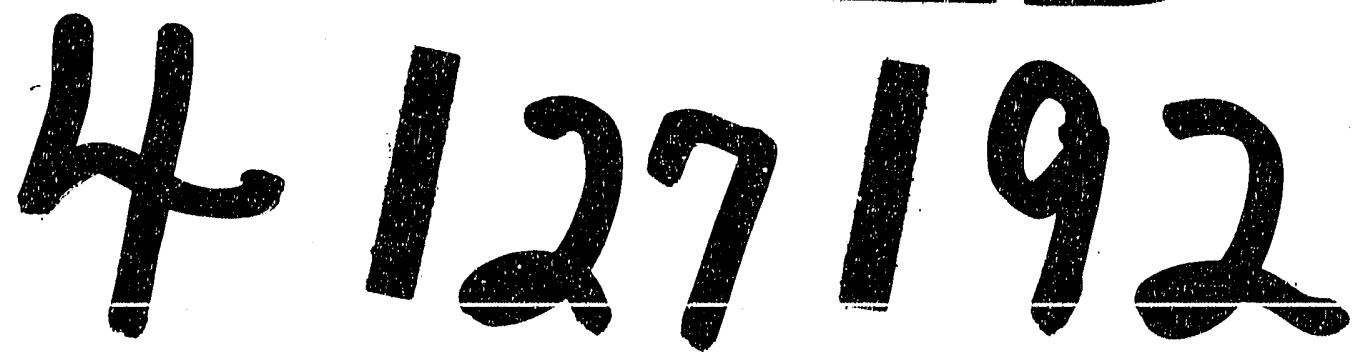


